\title{
Características clínicas e comorbidades associadas à mortalidade por insuficiência cardíaca em um hospital de alta complexidade na Região Amazônica do Brasil
}

\section{Clinical characteristics and comorbidities associated with heart failure mortality in a highly complex hospital in the Amazon Region of Brazil}

\author{
Weydder Tavares da Silva' (D), Milene Gouvêa Tyll2 (D), Ana Cristina Cardoso de Sousa Miranda ${ }^{3}$ (D), Giovanna Pontes \\ Moura $^{3}$ iD, Adriana de Oliveira Lameira Veríssimo ${ }^{3}$ iD \\ ' Universidade Federal do Pará, Faculdade de Medicina, Belém, Pará, Brasil \\ 2 Universidade da Amazônia, Belém, Pará, Brasil \\ ${ }^{3}$ Fundação Hospital de Clínicas Gaspar Vianna, Belém, Pará, Brasil
}

\begin{abstract}
RESUMO
OBJETIVOS: Identificar as características clínicas e socioepidemiológicas de pacientes com insuficiência cardíaca (IC) no atendimento de emergência em um hospital de alta complexidade na Região Amazônica do Brasil, assim como, as comorbidades associadas à mortalidade dos pacientes com IC descompensada. MATERIAIS E MÉTODOS: Estudo longitudinal e prospectivo, realizado em um hospital público de referência para emergência cardiológica na Região Norte do Brasil. Fizeram parte da pesquisa 125 pacientes com IC descompensada. RESULTADOS: Evidenciou-se que a principal etiologia da IC foi hipertensão arterial sistêmica $(39,2 \%)$, seguida de cardiomiopatia dilatada idiopática $(25,6 \%)$, sendo que a etiologia menos comum foi miocardite $(0,8 \%)$. A causa mais comum de descompensação foi a má adesão à terapia medicamentosa dos pacientes $(41,6 \%)$, seguida de infecções $(21,6 \%)$. Os principais sintomas apresentados pelos pacientes foram dispneia $(96,0 \%)$ e edema de tornozelos bilateral $(72,0 \%)$. Quanto à mortalidade, não houve relação estatisticamente significativa entre o perfil hemodinâmico à admissão com a mortalidade dos pacientes com IC descompensada $(p=0,4559)$. CONCLUSÃO: Com relação às características clínicas, o principal perfil hemodinâmico encontrado foi o frio e congesto, considerado o de pior prognóstico. A má adesão à terapia medicamentosa e infecções foram as principais causas de descompensação, apesar de não ter sido encontrada correlação estatisticamente significante das mesmas com a mortalidade. Assim, deve-se orientar o paciente quanto à adesão correta ao acompanhamento do tratamento medicamentoso e aos sintomas da IC congestiva, para que procure ajuda em tempo hábil e, com isso, melhore sua qualidade de vida.
\end{abstract}

Palavras-chave: Insuficiência Cardíaca; Prognóstico; Perfil de Saúde; Cardiopatias.

\begin{abstract}
OBJECTIVES: To identify the clinical and socio-epidemiological characteristics of patients with heart failure (HF) in emergency care at a highly complex hospital in the Amazon Region of Brazil, as well as the comorbidities associated with the mortality of patients with decompensated HF. MATERIALS AND METHODS: Longitudinal and prospective study, carried out in a public hospital of reference for cardiological emergency in the Northern Region of Brazil. This study included 125 patients with decompensated HF. RESULTS: The main etiology of HF was systemic arterial hypertension (39.2\%), followed by idiopathic dilated cardiomyopathy $(25.6 \%)$, with the least common etiology being myocarditis $(0.8 \%)$. The most common cause of decompensation was patients' poor adherence to drug therapy (41.6\%), followed by infections (21.6\%). The main symptoms presented by the patients were dyspnea (96.0\%) and bilateral ankle edema (72.0\%). As for mortality, there was no statistical significance between the hemodynamic profile at admission and the mortality of patients with decompensated HF ( $p=0.4555)$. CONCLUSION: Regarding clinical characteristics, the main hemodynamic profile found was cold and congestion, considered the worst prognosis. Poor adherence to drug therapy and infections were the main causes of decompensation, although no statistically significant correlation was found with mortality. Thus, patients should be instructed on the correct adherence to drug treatment and how to identify congestive HF symptoms, so that they can seek help in time and thereby improve their quality of life.
\end{abstract}

Keywords: Heart Failure; Prognosis; Health Profile; Heart Diseases.

\section{Correspondência / Correspondence:}

Adriana de Oliveira Lameira Veríssimo

Rua Rodolfo Chermont, 632. Bairro: Marambaia. CEP: 66615-170 - Belém, Pará, Brasil

E-mail: adylameira@gmail.com 


\section{INTRODUÇÃO}

A insuficiência cardíaca (IC) é definida como uma incapacidade do coração em bombear sangue adequadamente para todo o corpo para suprir as necessidades metabólicas do mesmo'.

Apesar dos avanços no manejo da IC nas últimas décadas, essa doença ainda continua sendo um problema de saúde pública, por apresentar alta prevalência e permanecer com resultados insatisfatórios, mesmo com o tratamento otimizado ${ }^{2}$. Acredita-se que a IC seja a via final das cardiopatias de diversas etiologias $^{3}$, e sua mortalidade pode alcançar até $50 \%$ nos cinco anos que se sucedem ao diagnóstico ${ }^{4}$.

Foi demonstrado que as doenças cardiovasculares estão entre as principais causas de morte no mundo. Por sua alta prevalência, a IC é causa frequente de internação hospitalar e de mortalidade intra-hospitalar ${ }^{5}$.

Essa enfermidade acomete principalmente indivíduos acima de 65 anos de idade, com prevalência maior em doentes renais crônicos e com fibrilação atrial ${ }^{6}$. Dentre as características clínicas associadas à IC, estudos mostraram que é comum o acometimento de infecções pulmonares e urinárias, o que ocasiona um aumento da mortalidade em pacientes internados ${ }^{7,8}$.

Em 2007, no Brasil, foram registradas 293.473 internações por IC, representando 2,59\% das internações hospitalares, a segunda causa clínica mais frequente, consumindo $3,05 \%$ do total de recursos financeiros?. Estudos realizados nas Regiões Sul e Centro-Oeste do Brasil mostraram que a sobrevida dos pacientes recém-diagnosticados com IC, no seguimento de 48 meses, foi significativamente reduzida ${ }^{10}$. Já na Região Norte, há carência de estudos que demonstrem o impacto da IC na mortalidade dessa população.

Devido à IC ser uma doença de abrangência mundial e apresentar índices elevados de morbidade e mortalidade ${ }^{11}$, este estudo objetivou identificar as características clínicas e socioepidemiológicas de pacientes com IC atendidos no serviço de emergência cardiológica de um hospital de referência na Região Amazônica do Brasil, assim como investigar possíveis fatores associados à mortalidade dos pacientes com IC descompensada.

\section{MATERIAIS E MÉTODOS}

Trata-se de um estudo descritivo, longitudinal prospectivo, conduzido na Divisão de Pesquisa da Fundação Hospital de Clínicas Gaspar Vianna (FHCGV) em Belém, no estado do Pará, que incluiu 125 pacientes com diagnóstico de IC descompensada, atendidos no serviço de emergência da instituição de 2014 a 2015, os quais foram submetidos a segmento durante três, seis e 12 meses a partir da inclusão no estudo. A FHCGV é o único hospital público da Região Norte referência para emergências cardiológicas, com atendimento exclusivo pelo Sistema Único de Saúde (SUS).

Para diagnosticar a IC, foram utilizados os critérios de Boston, descritos por Carlson et al.,
1985 (Quadro 1) 12, os quais são validados e de fácil aplicação. A pontuação acima de sete é considerada critério para o diagnóstico de IC definitiva.

\begin{tabular}{|c|c|}
\hline Critérios & Pontuação \\
\hline \multicolumn{2}{|l|}{ I. História } \\
\hline Dispneia em repouso & 4 \\
\hline Ortopneia & 4 \\
\hline Dispneia paroxística noturna & 3 \\
\hline Dispneia ao deambular & 2 \\
\hline Dispneia ao subir escadas & 1 \\
\hline \multicolumn{2}{|l|}{ II. Exame físico } \\
\hline \multicolumn{2}{|l|}{ Alteração na frequência cardíaca } \\
\hline $91-110 \mathrm{bmp}$ & 1 \\
\hline$>110 \mathrm{bmp}$ & 2 \\
\hline \multicolumn{2}{|l|}{ Distensão venosa jugular } \\
\hline$>6 \mathrm{~cm} \mathrm{H} 2 \mathrm{O}$ & 2 \\
\hline $\begin{array}{l}>6 \mathrm{~cm} \mathrm{H} 2 \mathrm{O} \text { mais edema ou } \\
\text { hepatomegalia }\end{array}$ & 3 \\
\hline \multicolumn{2}{|l|}{ Crepitantes pulmonares } \\
\hline Basais & 1 \\
\hline$>$ basais & 2 \\
\hline Sibilos & 3 \\
\hline Galope B3 & 3 \\
\hline \multicolumn{2}{|l|}{ III. Radiografia de tórax } \\
\hline Edema pulmonar alveolar & 4 \\
\hline Edema intersticial alveolar & 3 \\
\hline Derrame pleural bilateral & 3 \\
\hline Índice cardiotorácico > 0.50 & 3 \\
\hline Linhas B de Kerley & 2 \\
\hline
\end{tabular}

Fonte: Adaptado de Marantz et al. ${ }^{12}, 1988$.

Quadro 1 - Critérios de Boston para diagnosticar IC

Foram excluídos do estudo os pacientes submetidos a procedimentos de revascularização do miocárdio (angioplastia ou cirurgia) no mês anterior, a contar daquela internação, e pacientes que apresentavam sinais de IC secundária a um quadro de sepse.

Os dados obtidos por meio de entrevista e busca nos prontuários consistiram em características epidemiológicas (sexo, idade, raça/cor, escolaridade, renda) e clínicas (etiologia da IC, causa da descompensação da IC, sinais/sintomas apresentados na admissão, perfil hemodinâmico apresentado na admissão, comorbidades). As informações foram compiladas em um banco de dados no programa Microsoft Excel 2007, a partir do qual foram elaborados tabelas e gráficos. Foi realizada a análise estatística descritiva e, para a análise de significância, foram utilizados os testes qui-quadrado de Pearson, exato de Fisher e $G$, com nível $\alpha=0,05$ (5\%) e intervalo de confiança de $95 \%$, além da análise de sobrevida pela curva de Kaplan-Meier, por meio do software BioEstat v5.3.

Este estudo foi aprovado pelo Comitê de Ética em Pesquisa FHCGV, sob o parecer $n^{\circ}$ 1.553.691, 
em 16 de fevereiro de 2011. Os dados foram coletados da ficha de registro individual, a partir do prontuário do paciente, e por meio de entrevista, após assinatura do termo de consentimento livre e esclarecido.

\section{RESULTADOS}

Dos 125 pacientes incluídos neste estudo, $63,2 \%$ (79) eram do sexo feminino, $57,6 \%$ (72) se autodeclararam da raça/cor parda, 61,6\% (77) tinham o ensino fundamental completo ou incompleto e $52,8 \%$ (66) afirmaram ter como renda até um salário mínimo mensal (Tabela 1).

Tabela 1 - Características sociodemográficas dos pacientes com IC descompensada em um hospital de alta complexidade da Região Amazônica brasileira, de 2014 a 2015

\begin{tabular}{|c|c|c|}
\hline Características & $N=125$ & $\%$ \\
\hline \multicolumn{3}{|l|}{ Sexo } \\
\hline Masculino & 46 & 36,8 \\
\hline Feminino & 79 & 63,2 \\
\hline Idade & \multicolumn{2}{|c|}{$59,7^{\dagger} \pm 17,9^{\ddagger}$} \\
\hline \multicolumn{3}{|l|}{ Raça } \\
\hline Branca & 34 & 27,2 \\
\hline Negra & 14 & 11,2 \\
\hline Amarela & 1 & 0,8 \\
\hline Parda & 72 & 57,6 \\
\hline Indígena & 3 & 2,4 \\
\hline Não informado & 1 & 0,8 \\
\hline \multicolumn{3}{|l|}{ Escolaridade } \\
\hline Não alfabetizado & 31 & 24,8 \\
\hline Fundamental incompleto & 67 & 53,6 \\
\hline Fundamental completo & 10 & 8,0 \\
\hline Ensino médio incompleto & 5 & 4,0 \\
\hline Ensino médio completo & 5 & 4,0 \\
\hline Ensino superior incompleto & 3 & 2,4 \\
\hline Ensino superior completo & 3 & 2,4 \\
\hline Não informado & 1 & 0,8 \\
\hline \multicolumn{3}{|l|}{ Renda familiar (salário mínimo*) } \\
\hline$\leq 1$ & 66 & 52,8 \\
\hline$>1 a \leq 2$ & 50 & 40,0 \\
\hline$>2 a \leq 5$ & 8 & 6,4 \\
\hline$>5 a \leq 10$ & - & - \\
\hline$>10$ & - & - \\
\hline Não informado & 1 & 0,8 \\
\hline
\end{tabular}

* Salário mínimo equivalente a $\mathrm{R} \$ 998,00 ;{ }^{\dagger}$ Média de idade; ${ }^{\ddagger}$ Desvio padrão; Sinal convencional utilizado: - Dado numérico igual a zero, não resultante de arredondamento.
Quanto às etiologias da IC, 39,2\% (49) dos pacientes referiram hipertensão; 25,6\% (32), cardiomiopatia dilatada idiopática; e 13,6\% (17), causa isquêmica. Como fator desencadeante da descompensação da $\mathrm{IC}$, observou-se que a má adesão medicamentosa foi o principal gatilho para 41,6\% (52) dos pacientes, e infecções foi a segunda causa, com $21,6 \%$ (27) (Tabela 2).

Tabela 2 - Características clínicas dos pacientes com IC descompensada de um hospital de alta complexidade da Região Amazônica brasileira, de 2014 a 2015

\begin{tabular}{lcc}
\hline \multicolumn{1}{c}{ Características clínicas } & $\mathrm{N}=125$ & $\%$ \\
\hline Etiologias da IC & 49 & 39,2 \\
Hipertensiva & 32 & 25,6 \\
Cardiomiopatia dilatada idiopática & 17 & 13,6 \\
Isquêmica & 16 & 12,8 \\
Valvopatia & 8 & 6,4 \\
Cardiotoxicidade por quimioterápicos & 2 & 1,6 \\
Doença de Chagas & 1 & 0,8 \\
Miocardite & & \\
Causas da descompensação da IC & 52 & 41,6 \\
Má adesão à terapia medicamentosa & 27 & 21,6 \\
Infecções & 16 & 12,8 \\
Má adesão à terapia não medicamentosa & 16 & 12,8 \\
Arritmia & 16 & 12,8 \\
Evolução de valvopatia & 11 & 8,8 \\
Outras & &
\end{tabular}

Sinais/sintomas na admissão

Dispneia

12096,0

Edema de tornozelos bilateral $\quad 90 \quad 72,0$

Crepitações pulmonares

80

Turgência jugular

64,8

Hepatomegalia

31,2

22,4

Perfil hemodinâmico da IC na admissão

Frio e congesto

Quente e congesto

Frio e seco

Quente e seco

Não informado

Comorbidades

Hipertensão

Diabetes

Dislipidemia

Insuficiência renal crônica

Doença pulmonar obstrutiva crônica

Acidente vascular encefálico

Infarto agudo do miocárdio

Arritmia

Outras 
Com relação à sintomatologia, $96,0 \%$ (120) dos pacientes apresentaram dispneia; $72,0 \%$ (90), edema de tornozelos; e 64,8\% (81), crepitações pulmonares. O perfil hemodinâmico mais presente foi - frio e congesto, referenciado por $33,6 \%$ (42) dos pacientes. As comorbidades hipertensão (58,4\%; 73), diabetes $(34,4 \% ; 43)$ e dislipidemia $(27,2 \% ; 34)$ foram as mais prevalentes (Tabela 2).

Não houve relação estatisticamente significativa entre o perfil hemodinâmico na admissão com a mortalidade dos pacientes portadores de IC descompensada (Tabela 3), $p=0,4559$. Foi também analisada a relação das principais causas de descompensação da IC com a mortalidade (Tabela 4) e em nenhuma delas foi encontrada diferença significativa. Na curva de sobrevida geral dessa população verificou-se que a probabilidade de sobrevida geral acumulada foi de 59,5\%, 50,3\% e $48,2 \%$ após três, seis e 12 meses de seguimento, respectivamente (Figura 1).

A congestão pulmonar esteve presente na admissão em 94 pacientes $(75,2 \%)$ não apenas como sintoma (dispneia), mas também como sinal durante a admissão (ao exame de imagem - radiografia de tórax) e mostrou relação significante para IC descompensada $(p<0,0001)$.

Tabela 3 - Associação entre perfil hemodinâmico e mortalidade dos pacientes com IC descompensada de um hospital de alta complexidade da Região Amazônica brasileira, de 2014 a 2015

\begin{tabular}{lrrrrr}
\hline \multicolumn{1}{c}{ Perfil } & \multicolumn{2}{c}{ Óbitos } & \multicolumn{2}{c}{ Vivos } & p-valor* \\
hemodinâmico & N & $\%$ & \multicolumn{1}{c}{$N$} & $\%$ & \\
\hline Quente e seco & 5 & 9,8 & 6 & 8,1 & \\
Quente e congesto & 15 & 29,4 & 26 & 35,1 & 0,4559 \\
Frio e congesto & 21 & 41,2 & 21 & 28,4 & \\
Frio e seco & 10 & 19,6 & 21 & 28,4 & \\
\hline Total & 51 & 100,0 & 74 & 100,0 & \\
\hline
\end{tabular}

* Teste G.

Tabela 4 - Relação entre as principais causas de descompensação e mortalidade da IC de um hospital de alta complexidade da região Amazônica, nos anos de 2014 e 2015

\begin{tabular}{|c|c|c|c|c|c|}
\hline \multirow{2}{*}{$\begin{array}{c}\text { Causas de } \\
\text { descompensação de IC }\end{array}$} & \multicolumn{2}{|c|}{ Óbitos } & \multicolumn{2}{|c|}{ Vivos } & \multirow{2}{*}{ p-valor } \\
\hline & $N$ & $\%$ & $\mathrm{~N}$ & $\%$ & \\
\hline $\begin{array}{l}\text { Má adesão à terapia } \\
\text { medicamentosa }\end{array}$ & 23 & 44,2 & 29 & 55,8 & $0,8775^{*}$ \\
\hline Infecções & 10 & 37,0 & 17 & 63,0 & $0,5344^{*}$ \\
\hline $\begin{array}{l}\text { Má adesão à terapia } \\
\text { não medicamentosa }\end{array}$ & 5 & 31,2 & 11 & 68,8 & $0,6125^{*}$ \\
\hline Arritmia & 8 & 50,0 & 8 & 50,0 & $1,0000^{+}$ \\
\hline Evolução de valvopatia & 10 & 62,5 & 6 & 37,5 & $0,1809^{*}$ \\
\hline Outras & 3 & 27,3 & 8 & 72,7 & $0,3407^{\dagger}$ \\
\hline
\end{tabular}

* Teste qui-quadrado; ${ }^{\dagger}$ Teste exato de Fisher.

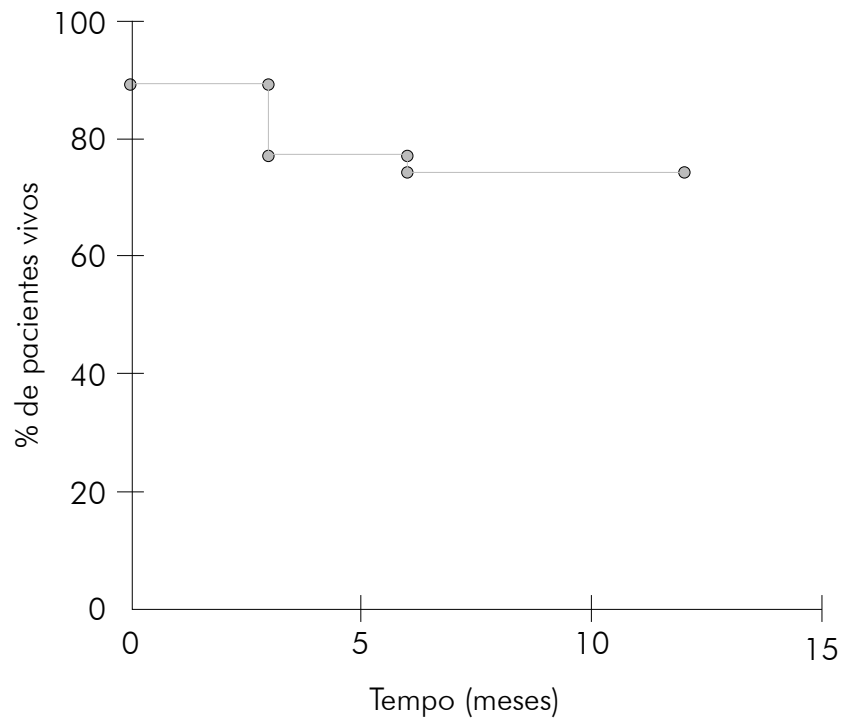

Figura 1 - Probabilidade de sobrevida geral acumulada dos pacientes internados com IC descompensada em um hospital de alta complexidade da Região Amazônica brasileira, de 2014 a 2015

\section{DISCUSSÃO}

A população com IC descompensada incluída nesta pesquisa se assemelha à população nacional e internacional com essa patologia, em relação ao sexo feminino ser 0 mais acometido pela doença ${ }^{13}$. Em um estudo com base nos dados do registro BREATHE, onde estavam incluídas todas as Regiões do Brasil, foi observado que o sexo feminino compreendia 60\% dessa população ${ }^{13}$. O registro Acute Decompensated Heart Failure National Registry (ADHERE), o maior registro internacional sobre IC, desenvolvido nos EUA, obteve resultado semelhante, com $52 \%$ da população correspondente ao sexo feminino ${ }^{14}$; no entanto, há estudos nos quais essa população difere ${ }^{15}$. Alguns autores defendem que essa prevalência do sexo feminino se justifica pela maior procura das mulheres pelos serviços de saúde ${ }^{3}$.

Pacientes com idade a partir de 60 anos estão presentes na maioria dos estudos sobre IC descompensada $13,14,15,16$. A média de idade, no presente estudo, foi de 59,7 (com variação de $\pm 17,9$ ). Estudos conduzidos na Austrália e na Colômbia encontraram uma média de idade ainda maior, em torno de $71 \operatorname{anos}^{17,18}$. Esses achados estão em concordância com o Framingham Heart Study' ${ }^{19}$, que demonstrou que a idade mais predisponente para o aparecimento da IC é a partir dos 60 anos, uma vez que o próprio processo de envelhecimento cardíaco, além das comorbidades muitas vezes mal conduzidas, favorecem o surgimento da doença ${ }^{20}$.

Neste estudo, a maioria dos indivíduos apresentou baixo grau de escolaridade e baixa renda familiar. Essas características socioepidemiológicas são conhecidas como fatores de risco e agravantes tanto para o desenvolvimento de IC quanto para a readmissão hospitalar ${ }^{21}$.

Sabe-se que a maioria dos casos de IC tem como etiologias principais a doença arterial coronariana, 
que se configura na cardiomiopatia isquêmica, a hipertensão arterial sistêmica e as valvopatias ${ }^{22}$. A hipertensão arterial sistêmica, principal causa de IC relatada neste estudo, é conhecidamente um importante fator de risco para doenças cardíacas e costuma ocorrer mais em mulheres, enquanto, nos homens, prevalece a doença arterial coronariana ${ }^{19,20,21}$.

As internações por descompensação da IC constituem um importante custo à saúde pública, cujas causas predominantes são a má adesão ao tratamento medicamentoso, a ingestão excessiva de sal e água, além de infecções e arritmias $^{23}$. No presente estudo, a má adesão à terapia medicamentosa $(41,6 \%)$ e infecções $(21,6 \%)$ foram as principais causas de descompensação, o que já foi evidenciado em outros estudos no Brasil ${ }^{13,24}$.

A má adesão está associada a diversos fatores e, portanto, não é de responsabilidade exclusiva do paciente. Um importante fator de risco para a má adesão medicamentosa é a idade, ressaltando que os pacientes com IC descompensada tendem a apresentar idade mais avançada. Em pacientes com IC é fundamental, durante a avaliação clínica, a busca por infecções, pois esse é um fator precipitante da descompensação que, além de frequente, está associado à gravidade ${ }^{25}$.

No estudo BREATHE ${ }^{13}$, o perfil hemodinâmico mais encontrado na admissão foi o quente e congesto $(67,4 \%)$, enquanto na amostra deste estudo prevaleceu o perfil frio e congesto, com $33,6 \%$ dos casos. $\bigcirc$ perfil quente e congesto configura como o mais frequente, enquanto que o perfil frio e congesto é considerado como 0 de pior prognóstico ${ }^{26}$. Os relatos dos pacientes, durante as entrevistas da pesquisa, sugerem que a demora em procurar o serviço de saúde esteja relacionada pela dificuldade de acesso ou mesmo pela má orientação quanto aos primeiros sinais/sintomas de descompensação da IC.

As manifestações clínicas mais frequentes neste estudo foram os sintomas congestivos: 96,0\% apresentaram dispneia; $72,0 \%$, edema de tornozelos bilateral; e 64,8\%, crepitações pulmonares. Inclusive, a congestão pulmonar esteve presente não só como sintoma (dispneia), mas também como sinal durante a admissão (ao exame de imagem - radiografia de tórax). Esses dados estão de acordo com os encontrados na literatura ${ }^{27,28}$. Os sinais/sintomas de IC descompensada, como os congestivos, constituem evidências clínicas de pacientes com alterações no sistema cardiovascular e são considerados características definidoras de IC $\mathrm{IC}^{20,29}$.

Quanto às comorbidades, foram encontradas hipertensão arterial sistêmica (58,4\%), diabetes (34,4\%) e dislipidemia $(27,2 \%)$ como as mais frequentes. Dados do estudo $\mathrm{EPICA}^{30}$ - Epidemiologia da IC - mostraram, como comorbidades mais frequentes presentes em pacientes com IC descompensada, tanto em serviços públicos quanto em privados, hipertensão arterial sistêmica, diabetes e doença pulmonar obstrutiva crônica. Outros estudos ${ }^{31,32}$ também mostraram a hipertensão arterial sistêmica como principal comorbidade e fator de risco para o desenvolvimento da doença.

Não houve relação entre o perfil hemodinâmico na admissão com a mortalidade dos pacientes com IC descompensada. Mesmo tendo demonstrado uma população de pacientes internados com perfil mais grave (frio e congesto), isso não significa que os pacientes que mais evoluíam a óbito encontravam-se nesse perfil hemodinâmico. De acordo com outros estudos 33,34 , a maioria dos pacientes com IC morre antes de chegar ao estágio $D$ da progressão da doença, ou seja, o estágio mais grave da IC, caracterizado como IC refratária ao tratamento clínico e que requer intervenção especializada', devido ao elevado risco de morte súbita ${ }^{35}$.

Com relação à classificação funcional da IC da New York Heart Association', que se baseia na gravidade dos sintomas apresentados, variando de I-IV, ausência de sintomas até a presença de sintomas mesmo em repouso, os pacientes com IC de início recente dos sintomas apresentavam uma boa curva de sobrevida; porém, os de classes funcionais III e IV tiveram o risco cardiovascular maior, quando comparados aos pacientes com classe funcional $1{ }^{10,19}$.

Quanto às principais causas de descompensação da IC associadas à mortalidade, não foi encontrada correlação estatisticamente significativa, mesmo tendo um número maior de pacientes que descompensaram por má adesão medicamentosa.

Sabe-se que alguns medicamentos importantes no manejo da IC não são ofertados pelo SUS e, mesmo que fossem, ainda haveria o inconveniente posológico, devido a alguns medicamentos terem que ser administrados em doses fracionadas durante o dia, dificultando a adesão, o que pode ser intensificado pelo problema da polifarmácia ${ }^{36,37}$. Além disso, há menos adesão ao tratamento não farmacológico, o qual é um importante componente no manejo da IC, mesmo quando a severidade da doença limita a atividade física dos pacientes ${ }^{38}$.

A sobrevida dos pacientes hospitalizados por IC descompensada foi de 67,4\% em 12 meses, taxa considerada baixa, principalmente considerando a introdução de medicações modificadoras de mortalidade após os anos $2000^{39}$. No presente estudo, a probabilidade de sobrevida geral acumulada em 12 meses foi de 48,2\%, taxa ainda menor. Esse dado pode ser justificado pelas manifestações clínicas dos pacientes serem de maior gravidade na admissão e pela maior taxa de mortalidade durante a evolução. Em contrapartida, a maioria dos ensaios clínicos para - cálculo de sobrevida requer pacientes com IC estável antes de recrutá-los ${ }^{10,40,41}$, sendo então difícil a comparação das taxa de sobrevida.

Uma das limitações deste estudo foi a não apresentação dos valores de fração de ejeção do ventrículo esquerdo (FEVE), o que seria um dado importante para classificar os pacientes portadores de IC com fração de ejeção preservada (ICFEP) e IC com 
fração de ejeção reduzida (ICFER), pois não havia disponibilidade desses dados na admissão do paciente e nem durante a internação.

\section{CONCLUSÃO}

Neste estudo, realizado em um hospital de referência para emergência cardiológica na Região Norte, com atendimento $100 \%$ SUS, os pacientes com IC descompensada internam, em sua maioria, com perfil hemodinâmico frio e congesto, considerado o de pior prognóstico, resultando em uma taxa de sobrevida geral acumulada em 12 meses de 48,2\%. Com relação às causas de descompensação, encontrou-se que a má adesão medicamentosa e as infecções foram os principais motivos, apesar de não ter sido encontrada correlação estatisticamente significante dessas causas com a mortalidade. Assim, deve-se orientar os pacientes quanto à adesão correta ao acompanhamento do tratamento medicamentoso e aos sintomas da IC congestiva, para procurarem ajuda em tempo hábil e, com isso, melhorarem sua qualidade de vida.

De qualquer forma, apesar dos avanços que possibilitaram um melhor controle dos sintomas da doença, a IC permanece como uma síndrome de grande impacto socioeconômico e alta mortalidade hospitalar. É necessário orientar esses pacientes quanto aos achados iniciais de descompensação para que possam procurar a tempo os serviços de saúde, a fim de que não entrem na fase de pior prognóstico; e, sobretudo, uma vez na assistência, sempre atentar para a anamnese adequada, o que favorece o manuseio correto em qualquer fase da descompensação.

\section{APOIO FINANCEIRO}

As fontes de financiamento do presente estudo foram de origem privada.

\section{CONFLITOS DE INTERESSE}

Os autores declaram que não houve conflitos de interesses.

\section{CONTRIBUIÇÃO DOS AUTORES}

Todos os autores contribuíram com a idealização do estudo, a análise e a interpretação dos dados e com a redação do manuscrito, aprovando a versão final publicada. Declaram-se responsáveis pelo conteúdo integral do artigo, garantindo sua precisão e integridade.

\section{REFERÊNCIAS}

1 Comitê Coordenador da Diretriz de Insuficiência Cardíaca (BR). Diretriz brasileira de insuficiência cardíaca crônica e aguda. Arq Bras Cardiol. 2018;111(3):436-539.

2 Willis MS, Patterson C. Proteotoxicity and cardiac dysfunction - Alzheimer's disease of the heart? $\mathrm{N}$ Engl J Med. 2013 Jan;368(5):455-64.

3 Wang TJ. Living without heart failure: contemporary concepts in prevention. JACC Heart Fail. 2016 Dec;4(12):920-2.

4 Gaui EN, Klein CH, Oliveira GMM. Mortalidade por insuficiência cardíaca: análise ampliada e tendência temporal em três estados do Brasil. Arq Bras Cardiol. 2010 jan;94(1):55-61.

5 Barretto ACP, Del Carlo CH, Cardoso JN, Morgado PC, Munhoz RT, Eid MO, et al. Re- hospitalizações e morte por insuficiência cardíaca: índices ainda alarmantes. Arq Bras Cardiol. 2008;91 (5):335-41.

6 Barbosa RR, Nascimento CCR, Calil OA, Serpa RG, Corteletti A, Jacques TM, et al. Insuficiência cardíaca na população geriátrica: dados de um registro unicêntrico. Rev Soc Bras Clin Med. 2018 out-dez; 1 6(4):203-7.

7 Cardoso JN, Del Carlo CH, Oliveira Jr MT, Ochiai ME, Kalil Filho R, Barretto ACP. Infecção em pacientes com insuficiência cardíaca descompensada: mortalidade hospitalar e evolução. Arq Bras Cardiol. 2018 abr;1 10(4):364-70.

8 Formiga F, Moreno-Gonzalez R, Chivite D, Casado J, Escrihuela-Vidal F, Corbella X. Clinical characteristics and one-year mortality according to admission renal function in patients with a first acute heart failure hospitalization. Rev Port Cardiol. 2018 Feb;37(2): 159-65.

9 Reis MB, Dias MG, Bibanco MS, Lopes CT, Gea GN. Readmissão hospitalar por insuficiência cardíaca em um hospital de ensino do interior do Estado de São Paulo-SP. Medicina (Ribeirão Preto). 2015;48(2):138-42.

10 Rassi S, Barretto ACP, Porto CC, Pereira CR, Calaça BW, Rassi DC. Sobrevida e fatores prognósticos na insuficiência cardíaca sistólica com início recente dos sintomas. Arq Bras Cardiol. 2005 abr;84(4):309-13.

11 Weidmann ZM, Breidthardt T, Twerenbold R, Zûsli C, Nowak A, von Eckardstein A, et al. Prediction of mortality using quantification of renal function in acute heart failure. Int J Cardiol. 2015 Dec;201:650-7.

12 Marantz PR, Tobin JN, Wassertheil-Smoller S, Steingart RM, Wexler JP, Budner $N$, et al. The relationship between left ventricular systolic function and congestive heart failure diagnosed by clinical criteria. Circulation. 1988 Mar;77(3):607-12.

13 Albuquerque DC, Souza Neto JD, Bacal F, Rohde LEP, Bernadez-Pereira S, Berwanger $O$, et al. I Registro brasileiro de insuficiência cardíaca - aspectos clínicos, qualidade assistencial e desfechos hospitalares. Arq Bras Cardiol. 2015 jun; 104(6):433-42. 
14 Gripp EA, Tedeschi B, Sales ALF, Iso MA, Spineti PPM, Coloma $M$, et al. Os resultados dos registros internacionais de insuficiência cardíaca descompensada se aplicam aos pacientes brasileiros? Rev Soceri. 2009 mai-jun;22(3):165-9.

15 Stewart S, Carrington MJ, Horowitz JD, Marwick TH, Newton PJ, Davidson PM, et al. Prolonged impact of home versus clinic-based management of chronic heart failure: extended follow-up of a pragmatic, multicentre randomized trial cohort. Int J Cardiol. 2014 Jul;174(3):600-10.

16 Ahmad FS, Barg FK, Bowles KH, Alexander M, Goldberg LR, French $B$, et al. Comparing perspectives of patients, caregivers, and clinicians on heart failure management. J Card Fail. 2016 Mar;22(3):210-7.

17 Feola M, Lombardo E, Testa M, Avogadri E, Piccolo S, Vado A. Prognostic factors of mid-term clinical outcome in congestive heart failure patients discharged after acute decompensation. Arch Med Sci. 2012 Jul;8(3):462-70.

18 Zuluaga JSP, Carrasquilla KM, Granda AMV, Giraldo CIS, Ossa AMQ, Arenas EMC, et al. Predictores de hospitalización prolongada en pacientes con insuficiencia cardiaca aguda. Rev Colomb Cardiol. 2019 mar-abr;26(2):78-85.

19 Ho KKL, Pinsky JL, Kannel WB, Levy D. The epidemiology of heart failure: the Framingham Study. J Am Coll Cardiol. 1993 Oct;22(4 Suppl 1):A6-13.

20 Testani JM, Ter Maaten JM. Decongestion in acute heart failure: does the end justify the means? JACC Heart Fail. 2016 Jul;4(7):589-90.

21 Bittencourt M, Ribeiro FCV, Rocha RM, Albuquerque DC. Importância do diagnóstico etiológico na insuficiência cardíaca idiopática. Rev Bras Cardiol. 2014 mar-abr;27(2):128-30.

22 Castaño JCV, Hernández AA, Cabrera AC. Efectividad de la intervención terapéutica ambulatoria en adultos con insuficiencia cardíaca crónica. Rev Cubana Salud Publica. 2015 abr-jun;41 (2):253-67.

23 Passos VMA, Assis TD, Barreto SM. Hipertensão arterial no Brasil: estimativa de prevalência a partir de estudos de base populacional. Epidemiol Serv Saude. 2006 mar; 15(1):35-45.

24 Rabelo-Silva ER, Saffi MAL, Aliti GB, Feijó MK, Linch GFC, Saver JM, et al. Fatores precipitantes de descompensação da insuficiência cardíaca relacionados a adesão ao tratamento: estudo multicêntrico-EMBRACE. Rev Gaucha Enferm. 2018 out;39:e20170292.

25 Horwich TB, Fonarow GC. Prevention of heart failure. JAMA Cardiol. 2017 Jan;2(1):116.

26 Mangini S, Pires PV, Braga FGM, Bacal F. Insuficiência cardíaca descompensada. Einstein (São Paulo). 2013 jul-set;11 (3):383-91.
27 Kalogeropoulos AP, Georgiopoulou W, Murphy RA, Newman AB, Baver DC, Harris TB, et al. Dietary sodium content, mortality, and risk for cardiovascular events in older adults: the health, aging, and body composition (Health $A B C$ ) study. JAMA Intern Med. 2015;175(3):410-9.

28 Nuti SV, Qin L, Rumsfeld JS, Ross JS, Masoudi FA, Normand SLT, et al. Association of admission to veterans affairs hospitals vs non-veterans affairs hospitals with mortality and readmission rates among older men hospitalized with acute myocardial infarction, heart failure, or pneumonia. JAMA. 2016 Feb;315(6):582-92.

29 Redfield MM. Heart failure with preserved ejection fraction. N Engl J Med. 2016 Nov;375(19):1868-77.

30 Tavares LR, Victer $H$, Linhares JM, Barros CM, Oliveira MV, Pacheco LC, et al. Epidemiologia da insuficiência cardíaca descompensada em Niterói - Projeto EPICA - Niterói. Arq Bras Cardiol. 2004 fev;82(2): 121-4.

31 Nogueira PR, Rassi S, Corrêa KS. Perfil epidemiológico, clínico e terapêutico da insuficiência cardíaca em hospital terciário. Arq Bras Cardiol. 2010 set;95(3):392-8.

32 von Haehling S, Hasenfuß G, Anker SD. Diabetes and heart failure: sugared words prove bitter. J Am Coll Cardiol. 2016 Sep;68(13):1417-9.

33 Melo Neto AJ, Mosele F, Fuchs SC. Risk of heart failure with human immunodeficiency virus is greater than hypertension? Arch Intern Med. 2011 Oct;171(19):1773-4.

34 Whellan DJ, Goodlin SJ, Dickinson MG, Heidenreich PA, Jaenicke C, Stough WG, et al. End-of-life care in patients with heart failure. J Card Fail. 2014 Feb;20(2):121-34.

35 Kober L, Torp-Pedersen C, McMurray JJV, Gotzsche $O$, Levy $S$, Crijns $H$, et al. Increased mortality after dronedarone therapy for severe heart failure. $N$ Engl J Med. 2008 Jun;358(25):2678-87.

36 Sarwar CMS, Papadimitriou L, Pitt B, Pina I, Zannad F, Anker SD, et al. Hyperkalemia in heart failure. J Am Coll Cardiol. 2016 Oct;68(14):1575-89.

37 Nieminen MS, Dickstein K, Fonseca C, Serrano JM, Parissis J, Fedele F, et al. The patient perspective: quality of life in advanced heart failure with frequent hospitalisations. Int J Cardiol. 2015 Jul;191:256-64.

38 Hauptman PJ, Goff ZD, Vidic A, Chibnall JT, Bleske BE. Variability in retail pricing of generic drugs for heart failure. JAMA Intern Med. 2017 Jan;177(1):126-8.

39 Del Carlo CH, Cardoso JN, Ochia ME, Oliveira Jr MT, Ramires JAF, Pereira-Barretto AC. Variação temporal no prognóstico e tratamento da insuficiência cardíaca avançada - antes e após 2000. Arq Bras Cardiol. 2014 mai;102(5):495-504. 
40 Kaufman R, Azevedo VMP, Xavier RMA, Geller M, Chaves RBM, Castier MB. Insuficiência cardíaca: análise de 12 anos da evolução em internações hospitalares e mortalidade. Int J Cardiovasc Sci. 2015;28(4):276-81.
41 Bocchi EA, Braga FGM, Ferreira SMA, Rohde LEP, Oliveira WA, Almeida DR, et al. III Diretriz brasileira de insuficiência cardíaca crônica. Arq Bras Cardiol. 2009;93(1 supl 1):1-71.

Recebido em / Received: 5/8/2019

Aceito em / Accepted: 25/8/2020

Como citar este artigo / How to cite this article:

Silva WT, Tyll MG, Miranda ACCS, Moura GP, Veríssimo AOL. Características clínicas e comorbidades associadas à mortalidade por insuficiência cardíaca em um hospital de alta complexidade na Região Amazônica do Brasil. Rev Pan Amaz Saude. 2020;1 1:e202000449. Doi: http://dx.doi. org/10.5123/S2176-6223202000449 